\title{
A De-correlated Modified Code Tracking Loop for Synchronous Direct-Sequence Code-Division Multiple-Access Systems
}

\author{
W K Wong and $\mathrm{C} \mathrm{W}$ Leung \\ Electronic Engineering Department \\ City University of Hong Kong \\ 83 Tat Chee Avenue \\ Hong Kong
}

\begin{abstract}
A de-correlated modified code tracking loop (D-MCTL) is presented in this paper for synchronous direct-sequence code-division multiple-access (DS/CDMA) systems. In this paper, it is found that the $S$ curve of a traditional modified code tracking loop (MCTL) may be severely distorted; even the multiple access interference (MAI) is low at the ideal sampling instant. The proposed D-MCTL alleviates the effect of MAI at \pm 1 chip interval caused by interfering users and achieves a better $S$ curve with a zero timing offset. Computer simulations based on Gold code sequences are used to verify the results.
\end{abstract}

\section{INTRODUCTION}

A modified code tracking loop (MCTL) is proposed in [1]. It has better tracking performance and is less sensitive to gain imbalances compared with the traditional delay locked loop $[2,3]$ under single user conditions. However, both of them suffer from multiple access interference (MAI) due to the non-orthogonal signature of interfering users. Preliminary works have been done on the performance of traditional delay locked loop under MAI [4-8] and to the best of the authors' knowledge; no similar analysis has been performed for MCTL. In this paper, the static response of the loop, i.e. the $S$ curve, is used as the performance measure. It is found that an MCTL's $\boldsymbol{S}$ curve may be severely distorted; even the MAI is low at the ideal sampling instant. This low MAI may be achieved by various cancellation techniques or code selection. However, the strong MAI at \pm 1 chip interval not only creates timing offset bias but may also cause a stability problem. In this paper, the deterioration of the $S$ curve in an MCTL due to the MAI at \pm 1 chip interval is analyzed for a synchronous DS/CDMA system and a de-correlated MCTL (D-MCTL) is proposed to alleviate it. In a D-MCTL, the received signal correlates with the projection of de-correlating signals on the subspace orthogonal to the subspace spanned by the interfering signals. The MAI thus vanishes at a zero timing offset and produces a better $\boldsymbol{S}$ curve. Computer simulations based on Gold code sequences are used to compare the static performance of a traditional MCTL with a D-MCTL.

\section{TRANSMISSION MODEL FOR A DS/CDMA SYSTEM}

In this Paper, we focus on the influence of MAI, and neither fading nor other channel distortion will be addressed here.
The received signal for a $K$-user DS/CDMA system with BPSK modulation can be expressed as:

$$
r(t)=\sum_{k=1}^{K} \sqrt{2 P_{k}} b_{k}\left(t-\tau_{k}\right) c_{k}\left(t-\tau_{k}\right) \cos \left(\omega_{c} t+\boldsymbol{\theta}_{k}\right)+n(t)
$$

where subscript $k$ denotes the $k^{\text {th }}$ user.

o $P_{k}$ is the received power (assumed to be constant).

o $\quad b_{k}(t)=\sum_{m=-\infty}^{\infty} a_{k, m} p_{b}\left(t-m T_{b}\right)$ is the data signal, $a_{k, m}$ represents the binary sequence taking values \pm 1 with equal probability, $T_{b}$ represents the bit duration, and $p_{b}(t)$ is the rectangular bit waveform.

o $\quad c_{k}(t)=\sum_{l=1}^{N} u_{k, l} p_{c}\left[t-(l-1) T_{c}\right]$ is the signature waveform, $u_{k, l}$ is the code sequence taking values $\pm 1, T_{c}$ represents the chip duration, $p_{c}(t)$ is the rectangular chip waveform, and $N$ is the sequence length (short code is considered in this paper).

o $\quad \omega_{c}$ is the carrier frequency and $\boldsymbol{\theta}_{k}$ is the carrier phase.

o $n(t)$ is the Gaussian noise with double-sided power spectra density $N_{0} / 2$, which has the bandpass representation:

$$
n(t)=\sqrt{2}\left[n_{c}(t) \cos \left(\omega_{c} t\right)-n_{s}(t) \sin \left(\omega_{c} t\right)\right]
$$

$n_{c}(t)$ and $n_{s}(t)$ are independent, stationary, low-pass Gaussian processes with the same double-sided power spectra density $N_{0} / 2$.

All the delays are equal in a synchronous channel, i.e. $\tau_{1}=\tau_{2}$ $=\ldots=\tau_{\mathrm{k}}=\tau$. Without loss of generality, let us assume user 1 , i.e. $k=1$, is our desired user.

\section{AN MCTL IN DS/CDMA SYSTEMS}

The block diagram of a simplified MCTL is shown in Fig.1, where $\hat{\tau}$ is the estimated delay and $\Delta=T_{c} / 2$. Under normal conditions, the code self noise and modulation self noise can be ignored [9]. The equivalent error signal for the control loop can be separated into time independent and time dependent parts, as shown in the following: 
$e^{M}(t, \xi)=Q_{\text {static }}^{M}(\xi)+Q^{M}(t, \xi) \quad$ where $\xi=\tau-\hat{\tau}$

where superscript $M$ denotes the responses for a traditional MCTL.

Time independent signal $Q_{\text {static }}^{M}(\xi)$ :

$$
\begin{aligned}
Q_{\text {static }}^{M}(\xi)=D P_{1} R_{1,1}(\xi)\left[R_{1,1}(\xi-\Delta)-R_{1,1}(\xi+\Delta)\right] \\
+D \sum_{k=2}^{K} P_{k} R_{k, 1}(\xi)\left[R_{k, 1}(\xi-\Delta)-R_{k, 1}(\xi+\Delta)\right]
\end{aligned}
$$

$R_{i, j}(\xi \pm \Delta)=\overline{c_{i}(t-\tau) c_{j}(t-\hat{\tau} \pm \Delta)}$, where the over bar refers to statistical expectation.

$D=\overline{\left(b_{k}(t-\tau) \otimes l(t)\right)^{2}}$, where $\otimes$ refers to the convolution process and $l(t)$ is the baseband equivalent impulse response of BPF.

The first two terms in (1) denote the normal $\boldsymbol{S}$ curve characteristics of a traditional MCTL. The last summation terms correspond to the cross correlation from other users, or the so-called MAI. A non-zero value corresponds to a timing offset bias. Furthermore, if the overall MAI has a large positive value for $\xi<0$ and a large negative value for $\xi>0$, the slope of the $S$ curve may change its sign and enter into an unstable region. Assuming Gold code sequences are used, the worst-case scenario occurs when there exists large cross correlation values for $\left|R_{k, 1}\left(-T_{c}\right)\right|$ and $\left|R_{k, 1}\left(T_{c}\right)\right|$, $\operatorname{sgn}\left[R_{k, 1}\left(-T_{c}\right)\right]=-\operatorname{sgn}\left[R_{k, 1}\left(T_{c}\right)\right]$, and a low cross correlation value for $\left|R_{k, 1}(0)\right|$.

Time dependent signal $Q^{M}(t, \xi)$ :

The time dependent signal can be divided into three parts: signal X signal $\left(Q_{1}^{M}(t, \xi)\right)$, noise X noise $\left(Q_{2}^{M}(t, \xi)\right)$, and signal X noise $\left(Q_{3}^{M}(t, \xi)\right)$ :

$$
\begin{aligned}
& Q^{M}(t, \xi)=Q_{1}^{M}(t, \xi)+Q_{2}^{M}(t, \xi)+Q_{3}^{M}(t, \xi) \\
& Q_{1}^{M}(t, \xi)=\sum_{\substack{i=1 \\
i \neq j}}^{K} \sum_{j=1}^{K} \sqrt{P_{i} P_{j}} \tilde{b}_{i}(t) \tilde{b}_{j}(t) R_{i, 1}(\xi)\left[R_{j, 1}(\xi-\Delta)\right. \\
& \left.-R_{j, 1}(\xi+\Delta)\right] \cos \left(\boldsymbol{\theta}_{i}-\boldsymbol{\theta}_{j}\right) \ldots \ldots . \\
& Q_{2}^{M}(t, \xi)=N_{c 0}(t) N_{c \Delta}(t)+N_{s 0}(t) N_{s \Delta}(t) \\
& Q_{3}^{M}(t, \xi)=\sum_{i=1}^{K} \sqrt{P_{i} b_{i}}(t)\left\{N_{i \Delta}(t) R_{i, 1}(\xi)\right. \\
& \left.+N_{i 0}(t)\left[R_{i, 1}(\xi-\Delta)-R_{i, 1}(\xi+\Delta)\right]\right\}
\end{aligned}
$$

where $\tilde{b}_{k}(t)=b_{k}(t-\tau) \otimes l(t)$;

$$
\begin{aligned}
& N_{c 0}(t)=\left[n_{c}(t) c_{1}(t-\hat{\tau})\right] \otimes l(t) ; \\
& N_{s 0}(t)=\left[n_{s}(t) c_{1}(t-\hat{\tau})\right] \otimes l(t) ; \\
& N_{c \Delta}(t)=\left\{n_{c}(t)\left[c_{1}(t-\hat{\tau}-\Delta)-c_{1}(t-\hat{\tau}+\Delta)\right]\right\} \otimes l(t) ; \\
& N_{s \Delta}(t)=\left\{n_{s}(t)\left[c_{1}(t-\hat{\tau}-\Delta)-c_{1}(t-\hat{\tau}+\Delta)\right]\right\} \otimes l(t) ; \\
& N_{i 0}(t)=N_{c 0}(t) \cos \left(\boldsymbol{\Theta}_{i}\right)+N_{s 0}(t) \sin \left(\boldsymbol{\Theta}_{i}\right) ; i \in\{1,2, . ., K\} \\
& N_{i \Delta}(t)=N_{c \Delta}(t) \cos \left(\boldsymbol{\Theta}_{i}\right)+N_{s \Delta}(t) \sin \left(\boldsymbol{\Theta}_{i}\right) ; i \in\{1,2, . ., K\} .
\end{aligned}
$$

\section{A DE-CORRELATED MCTL IN DS/CDMA SYSTEMS}

The principle of a D-MCTL is to multiply the received signal $r(t)$ by the corresponding inverse cross correlation matrixes. The resultant signals are then orthogonal to the interferers such that the MAI vanishes at $\xi=0$. A similar structure has been proposed for the traditional delay lock loop [8]. The block diagram of a D-MCTL is shown in Fig.2, where the $s_{0}(t-\hat{\tau})$ and $s_{\Delta}(t-\hat{\tau})$ signals are:

$$
\begin{aligned}
& s_{0}(t-\hat{\tau})=\mathbf{A}_{0}^{\mathbf{T}} \mathbf{C}(t-\hat{\tau}) \ldots \ldots \ldots \ldots \ldots \ldots \ldots \ldots \ldots \ldots \ldots \ldots \ldots \\
& s_{\Delta}(t-\hat{\tau})=\mathbf{A}_{-}^{\mathbf{T}} \mathbf{C}_{-}(t-\hat{\tau})-\mathbf{A}_{+}^{\mathbf{T}} \mathbf{C}_{+}(t-\hat{\tau})
\end{aligned}
$$

where

$$
\begin{aligned}
& \mathbf{A}_{0}=\left[a_{10}, a_{20}, \ldots, a_{K 0}\right]^{T} \in \mathfrak{R}^{K x 1}, \\
& \mathbf{A}_{ \pm}=\left[a_{1 \pm}, a_{2 \pm}, \ldots, a_{K \pm}\right]^{T} \in \mathfrak{R}^{K x 1} \\
& \mathbf{C}(t-\hat{\tau})=\left[c_{1}(t-\hat{\tau}), c_{2}(t-\hat{\tau}), \ldots, c_{K}(t-\hat{\tau})\right]^{T} \in \mathfrak{R}^{K x 1}, \\
& \mathbf{C}_{ \pm}(t-\hat{\tau})=\left[c_{1}(t-\hat{\tau} \pm \Delta), c_{2}(t-\hat{\tau}), \ldots, c_{K}(t-\hat{\tau})\right]^{T} \in \mathfrak{R}^{K x 1}
\end{aligned}
$$

The corresponding outputs at the BPFs are:

$$
\begin{aligned}
y_{0}^{D}(t, \xi)=\sqrt{2} \mathbf{U}(t) \mathbf{R}_{0}(\xi) \mathbf{A}_{0}+\sqrt{2}[ & N_{c 0}^{D}(t) \cos (\boldsymbol{\omega} t) \\
& \left.-N_{s 0}^{D}(t) \sin (\boldsymbol{\omega} t)\right] \ldots . .
\end{aligned}
$$

where superscript $D$ denotes the responses for D-MCTL and

$$
\begin{array}{r}
\mathbf{U}(t)=\left[\sqrt{P_{1}} \tilde{b}_{1}(t) \cos \left(\omega t+\theta_{1}\right), \sqrt{P_{2} b_{2}}(t) \cos \left(\omega t+\theta_{2}\right),\right. \\
\left.\ldots, \sqrt{P_{K} b_{K}}(t) \cos \left(\omega t+\theta_{K}\right)\right] \in \mathfrak{R}^{1 x K}
\end{array}
$$

$\mathbf{R}_{0}(\xi)=\left[\begin{array}{cccc}R_{1,1}(\xi) & R_{1,2}(\xi) & . . & R_{1, K}(\xi) \\ R_{2,1}(\xi) & R_{2,2}(\xi) & . . & R_{2, K}(\xi) \\ . . & . . & . . & . . \\ R_{K, 1}(\xi) & R_{K, 2}(\xi) & & R_{K, K}(\xi)\end{array}\right] \in \Re^{K \times K}$

$N_{c 0}^{D}(t)=\left[n_{c}(t) s_{0}(t-\hat{\tau})\right] \otimes l(t)$

$N_{s 0}^{D}(t)=\left[n_{s}(t) s_{0}(t-\hat{\tau})\right] \otimes l(t)$ 


$$
\begin{aligned}
y_{\Delta}^{D}(t, \xi)=\sqrt{2} & {\left[\mathbf{U}(t) \mathbf{R}_{-}(\xi) \mathbf{A}_{-}-\mathbf{U}(t) \mathbf{R}_{+}(\xi) \mathbf{A}_{+}\right] } \\
& +\sqrt{2}\left[N_{c \Delta}^{D}(t) \cos (\boldsymbol{\omega} t)-N_{s \Delta}^{D}(t) \sin (\boldsymbol{\omega} t)\right] \ldots
\end{aligned}
$$

$\mathbf{R}_{ \pm}(\xi)=\left[\begin{array}{cccc}R_{1,1}(\xi \pm \Delta) & R_{1,2}(\xi) & . . & R_{1, K}(\xi) \\ R_{2,1}(\xi \pm \Delta) & R_{2,2}(\xi) & . . & R_{2, K}(\xi) \\ . . & . . & . . & . . \\ R_{K, 1}(\xi \pm \Delta) & R_{K, 2}(\xi) & & R_{K, K}(\xi)\end{array}\right] \in \Re^{K \times K}$

$N_{c \Delta}^{D}(t)=\left[n_{c}(t) s_{\Delta}(t-\hat{\tau})\right] \otimes l(t)$

$N_{s \Delta}^{D}(t)=\left[n_{s}(t) s_{\Delta}(t-\hat{\tau})\right] \otimes l(t)$

Let $\mathbf{D}_{\mathbf{0}}(\xi)=\mathbf{R}_{\mathbf{0}}(\xi) \mathbf{A}_{\mathbf{0}}=\left[d_{0}(\xi), d_{1}(\xi), \ldots, d_{K}(\xi)\right]^{T} \in \mathfrak{R}^{K x 1}$

It is obvious that $d_{0}(\xi)$ is the desired response and $d_{k}(\xi) \forall k \neq 1$ are the interfering terms. In order to cancel all the interfering terms at $\tau=\hat{\tau}$, i.e. $\xi=0$, let

$$
\mathbf{A}_{\mathbf{0}}=\mathbf{R}_{\mathbf{0}}^{-\mathbf{1}}(0)\left[d_{0}, 0,0, \ldots, 0\right]^{T}
$$

Similarly, $\mathbf{A}_{ \pm}=\mathbf{R}_{ \pm}^{-1}(0)[d, 0,0, \ldots, 0]^{T}$

where $d_{0}$ and $d$ are the normalization constants. The salient point of the above equations is that $\mathbf{A}_{\mathbf{0}}$ and $\mathbf{A}_{ \pm}$depend on the known or estimated cross correlation between users, but not on the received power of individual interfering users.

Adopting a similar approach to that used in the previous section, the equivalent error signal for the control loop can be separated into time independent and time dependent parts.

Time independent signal $Q_{\text {static }}^{D}(\xi)$ :

$$
Q_{\text {static }}^{D}(\xi)=\left[\mathbf{R}_{\mathbf{0}}(\xi) \mathbf{A}_{\mathbf{0}}\right]^{T} \mathbf{H}^{\prime}\left[\mathbf{R}_{-}(\xi) \mathbf{A}_{-}-\mathbf{R}_{+}(\xi) \mathbf{A}_{+}\right]
$$

where $\mathbf{H}^{\prime}$ is a diagonal matrix of data power,

i.e. $\mathbf{H}^{\prime}=\operatorname{diag}\left[P_{1} D, P_{2} D, P_{3} D, \ldots, P_{K} D\right] \in \mathfrak{R}^{K x K}$

Time dependent signal $Q^{D}(t, \xi)$ :

The time dependent signal can also be divided into three parts: signal X signal $\left(Q_{1}^{D}(t, \xi)\right)$, noise X noise $\left(Q_{2}^{D}(t, \xi)\right)$, and signal X noise $\left(Q_{3}^{D}(t, \xi)\right)$ :

i.e. $Q^{D}(t, \xi)=Q_{1}^{D}(t, \xi)+Q_{2}^{D}(t, \xi)+Q_{3}^{D}(t, \xi)$

$$
Q_{1}^{D}(\xi)=\left[\mathbf{R}_{\mathbf{0}}(\xi) \mathbf{A}_{\mathbf{0}}\right]^{T} \mathbf{H}\left[\mathbf{R}_{-}(\xi) \mathbf{A}_{-}-\mathbf{R}_{+}(\xi) \mathbf{A}_{+}\right]
$$

where $\mathbf{H}$ is the matrix of cross data power with all diagonal elements equal zero,

$$
\begin{aligned}
& \text { i.e. } \mathbf{H} \in \mathfrak{R}^{K x K},[\mathbf{H}]_{\mathrm{i}, \mathrm{j}}=\sqrt{P_{i} P_{j}} \widetilde{b}_{i}(t) \widetilde{b}_{j}(t) \cos \left(\boldsymbol{\Theta}_{\mathrm{i}}-\boldsymbol{\Theta}_{\mathrm{j}}\right) \quad \forall i \neq j \text {; } \\
& =0 \quad \forall i=j . \\
& Q_{2}^{D}(t, \xi)=N_{c 0}^{D}(t) N_{c \Delta}^{D}(t)+N_{s 0}^{D}(t) N_{s \Delta}^{D}(t) \\
& Q_{3}^{D}(t, \xi)=\mathbf{U}^{\prime}(t) \mathbf{N}_{\Delta}(t) \mathbf{R}_{\mathbf{0}}(\xi) \mathbf{A}_{\mathbf{0}} \\
& +\mathbf{U}^{\prime}(t) \mathbf{N}_{\mathbf{0}}(t)\left[\mathbf{R}_{-}(\xi) \mathbf{A}_{-}-\mathbf{R}_{+}(\xi) \mathbf{A}_{+}\right]
\end{aligned}
$$

where $\mathbf{U}^{\prime}(t)=\left[\sqrt{P_{1}} \widetilde{b}_{1}(t), \sqrt{P_{2}} \widetilde{b}_{2}(t), \ldots, \sqrt{P_{K}} \widetilde{b}_{K}(t)\right] \in \Re^{1 x K}$

$\mathbf{N}_{\Delta}(t)$ and $\mathbf{N}_{\mathbf{0}}(t)$ are the diagonal matrixes with $i^{\text {th }}$ elements equal:

$$
\begin{array}{ll}
N_{c \Delta}^{D}(t) \cos \left(\boldsymbol{\Theta}_{i}\right)+N_{s \Delta}^{D}(t) \sin \left(\boldsymbol{\Theta}_{i}\right) & \text { and } \\
N_{c 0}^{D}(t) \cos \left(\boldsymbol{\theta}_{i}\right)+N_{s 0}^{D}(t) \sin \left(\boldsymbol{\Theta}_{i}\right) & \text { respectively. }
\end{array}
$$

\section{Simulation Results}

The well-known Gold code sequences are used to simulate the performance of an MCTL and a D-MCTL. In this preliminary study, only the static response of the loop, or the so-called $\boldsymbol{S}$ curve, is used as the performance measure. It is too tedious to analyze the channel noise enhancement, i.e. $E\left\{\left[Q_{2}^{D}(t, \xi)\right]^{2}\right\} / E\left\{\left[Q_{2}^{M}(t, \xi)\right]^{2}\right\}$, or the variance of the tracking jitters by using Fokker-Plank equation [10] in this paper. The $\boldsymbol{S}$ curve is normalized to be \pm 0.5 at $\xi= \pm T_{c} / 2$ under no MAI condition. The effect of Gaussian noise has also not been taken into account at this stage because of its minority effect compared with MAI under most conditions.

All the simulation results are based on 2000 sets of random sequences of lengths 31 and 63 . The distributions of the positive and negative cross correlation peaks, after running through the whole set of the balanced Gold code sequences with various delays, are shown in Table 1. Apart from the preset cross correlation values and positions, the rest of the cross correlation terms of the simulations are distributed randomly and follow the statistics as described in Table 1; unless otherwise specified.

The effect of the near far problem is also included in the simulation, where the received power of half the interfering users is ' $\boldsymbol{n}-\boldsymbol{f}$ ' $\mathrm{dB}$ higher than that of the desired user. The first three sets of simulations that correspond to: 
$\begin{array}{lr}R_{i, 1}\left(-T_{c}\right)=-1+2^{\lfloor(n+2) / 2\rfloor}, & R_{i, 1}(0)=-1, \\ R_{i, 1}\left(+T_{c}\right)=-1-2^{\lfloor(n+2) / 2\rfloor}, & \forall i \neq 1,\end{array}$

sequence length $=2^{n}-1$,

are shown in Figs.3 to 5. In Fig.3 and Fig.4, it is clearly indicated that the traditional MCTL loses its tracking capability, but that the DMCTL is still able to maintain tracking and with a zero timing offset. In Fig.5, condition (a) refers to the normal distribution of cross correlation peaks as in Table 1; while condition (b) refers to reducing the number of positive and negative cross correlation peaks by half. It is shown that better bounds are obtained if the number of positive and negative correlation peaks is reduced.

Fig. 6 shows the simulation result that corresponds to:

$$
\begin{array}{lrl}
R_{i, 1}\left(-T_{c}\right)=-1-2^{\lfloor(n+2) / 2\rfloor}, & R_{i, 1}(0)=-1, \\
R_{i, 1}\left(+T_{c}\right)=-1, & \forall i \neq 1 .
\end{array}
$$

A timing offset is created and the $\boldsymbol{S}$ curve is in a critical stable condition for a traditional MCTL. The D-MCTL's resistance to MAI is demonstrated here, where a stable $\boldsymbol{S}$ curve with a zero timing offset is obtained. Fig. 7 shows that the simulation result corresponds to:

$$
\begin{array}{lrl}
R_{i, 1}\left(-T_{c}\right)=-1+2^{\lfloor(n+2) / 2\rfloor}, & R_{i, 1}(0)=-1, \\
R_{i, 1}\left(+T_{c}\right)=-1+2^{\lfloor(n+2) / 2\rfloor}, & \forall i \neq 1 .
\end{array}
$$

Here, the $\boldsymbol{S}$ curves for both the MCTL and the D-MCTL are degraded, and they achieve a similar performance.

\begin{tabular}{|c|c|c|}
\hline $\begin{array}{c}\text { Sequence Length } \\
N=2^{\mathrm{n}}-1\end{array}$ & $\begin{array}{c}\text { \% of positive cross } \\
\text { correlation peak } \\
\text { i.e. }-1+2^{\lfloor(n+2) / 2\rfloor}\end{array}$ & $\begin{array}{c}\text { \% of negative cross } \\
\text { correlation peak } \\
\left.\text { i.e. }-1-2^{\lfloor(n+2) / 2}\right\rfloor\end{array}$ \\
\hline 31 & 30.3 & 18.2 \\
\hline 63 & 15.4 & 9.2 \\
\hline
\end{tabular}

Table 1 Distribution of positive and negative cross correlation peaks for sequence lengths 31 and 63

\section{Conclusion}

In this paper, we demonstrate that a traditional MCTL may lose its tracking capability if strong MAI occurs at \pm 1 chip interval, and that even the MAI is low at the sampling instant. A de-correlated modified code tracking loop (D-MCTL) is proposed to alleviate such MAI and achieves a zero timing offset bias. Simulation results based on Gold code sequences show that there is a significant improvement on the $\boldsymbol{S}$ curve compared with that for the traditional MCTL. However, it should be noted that for large $K$ values, the $S$ curve of the D-MCTL may be seriously distorted, and the noise enhancement may also become significant. These issues need to be further investigated.

\section{REFERENCES}

[1] R A Yost and R W Boyd, "A modified PN code tracking loop: Its performance analysis and comparative evaluation," IEEE Trans. Commun., Vol 30, No 5, May 1982, pp.1027-1036.

[2] J J Spilker, "Delay lock tracking of binary signals," IEEE Trans. Space Electron. Telemetry, Vol 9, pp.1-8, Mar 1963.

[3] W J Gill, "A comparison of binary delay-lock loop implementations," IEEE Trans. Aerospace Electron. Syst., Vol 2, pp.415-424, July 1966.

[4] W Huang, I Andonovic, and M Nakagawa, "Code tracking of DS-CDMA systems in the presence of multi-user interference and additive noise," Proc IEEE International Symp. on SS Techniques and Appl., Vol 3, pp.843-847, 1998.

[5] W R Braun, "PN acquisition and tracking performance in DS/CDMA systems with symbol-length spreading sequences," IEEE Trans. Commun., Vol 45 No 12, pp.1595-1601, Dec 1997

[6] S E Hong, S Y Yoon, H S Lee, and J Ahn, "Performance Analysis of non-coherent delay-locked loop in multiple access interference," IEICE Trans. Commun., Vol E78-B, No 6, pp.935-941, Jun 1995.

[7] J Caffery, G L Stüber, "Effects of multiple -access iterference on the noncoherent delay lock loop," IEEE Trans. commun., Vol 48, No 12, pp.2109-2119, Dec 2000.

[8] W K Wong, "A de-correlated delay locked loop for direct sequence spread spectrum systems in a multi-user environment," accepted to be published in Proc IEEE MILCOM 2001, Oct 2001.

[9] M K Simon, "Noncoherent pseudonoise code tracking performance of spread spectrum receivers," IEEE Trans. Commun., Vol 25, No 3, Mar 1977, pp.327-345.

[10] H Meyr, "Delay-lock tracking of stochastic signals," IEEE Trans. Commun., Vol 24, No 3, pp.331-339, Mar 1976.

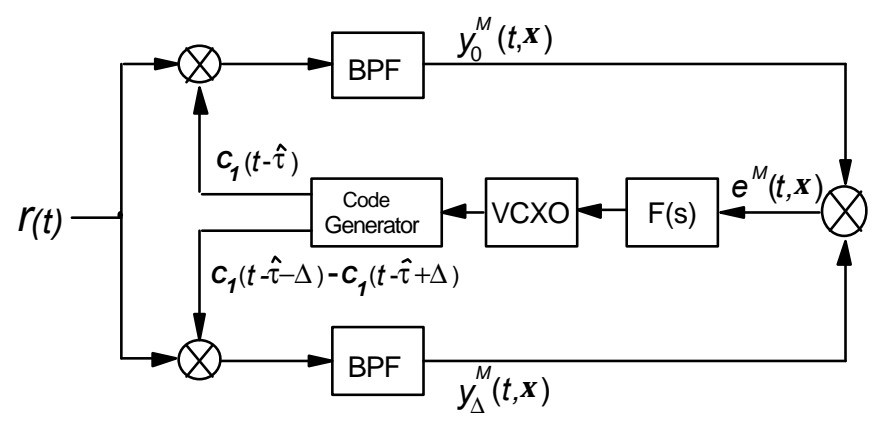

Fig.1 A Simplified Modified Code Tracking Loop (MCTL) 


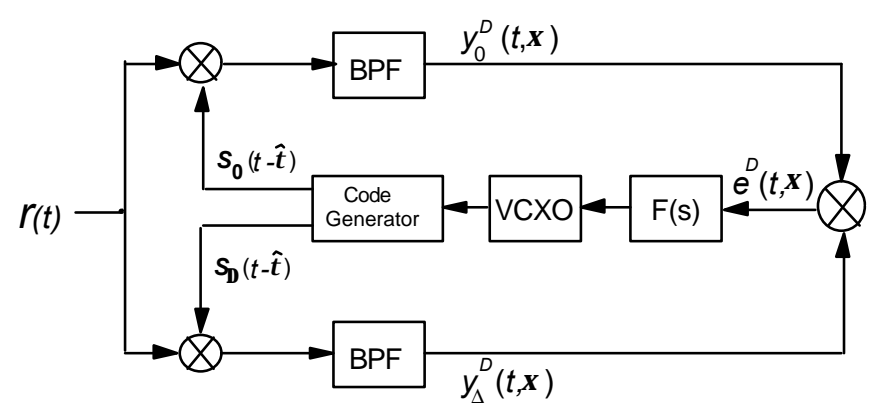

Fig.3 A De-correlated MCTL (D-MCTL).

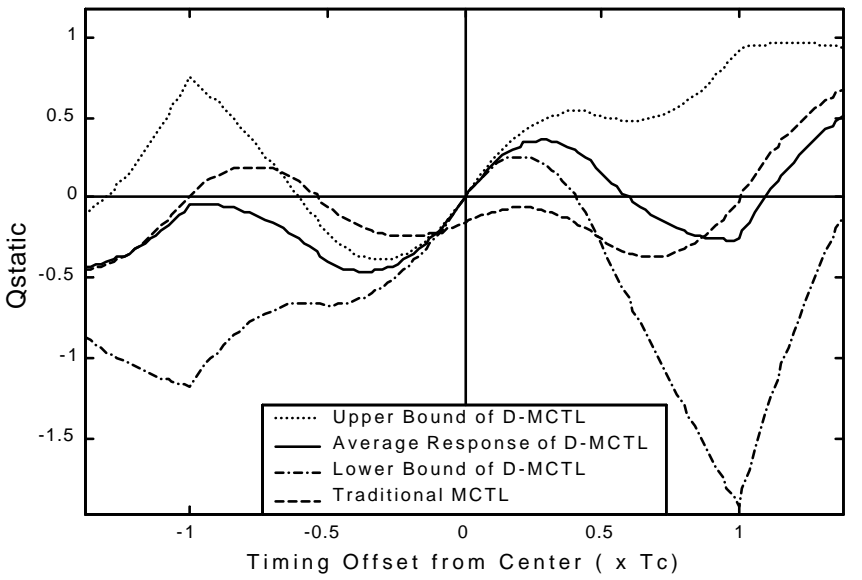

Fig.4 Sequence Length $=31$, no of users $=7, n-f=7 \mathrm{~dB}$, $R_{\mathrm{i}, 1}\left(-T_{C}\right)=+$ ve peak., $R_{\mathrm{i}, 1}\left(T_{\mathcal{C}}\right)=-$ ve peak

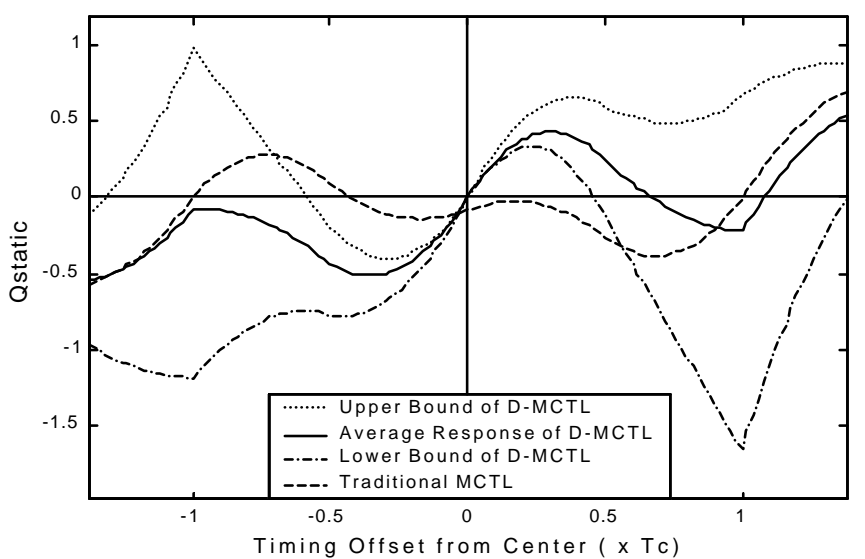

Fig.5 Sequence Length $=63$, no of users $=11, n-f=5 \mathrm{~dB}$, $R_{\mathrm{i}, 1}\left(-T_{\mathcal{C}}\right)=+$ ve peak., $R_{\mathrm{i}, 1}\left(T_{\mathcal{C}}\right)=-$ ve peak.

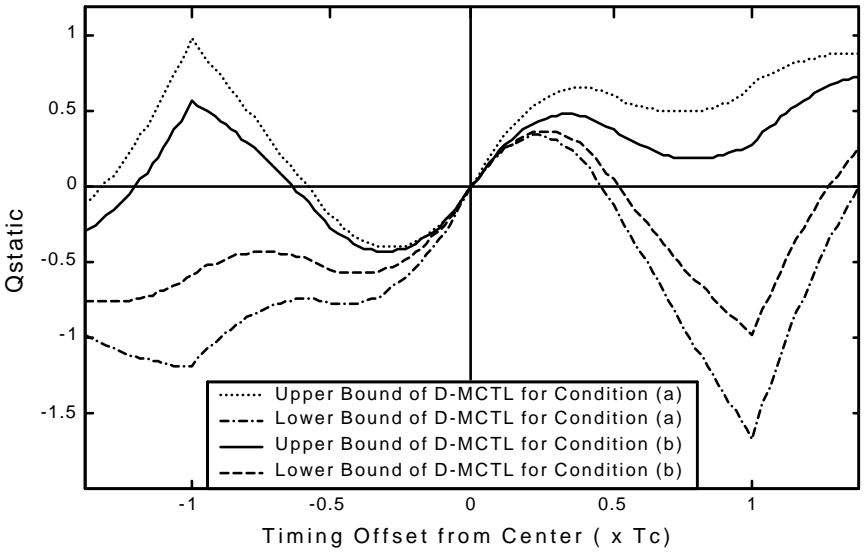

Fig. 5 Sequence Length $=63$, no of users $=11, n-f=5 \mathrm{~dB}$, a different distribution of cross correlation peaks is included.

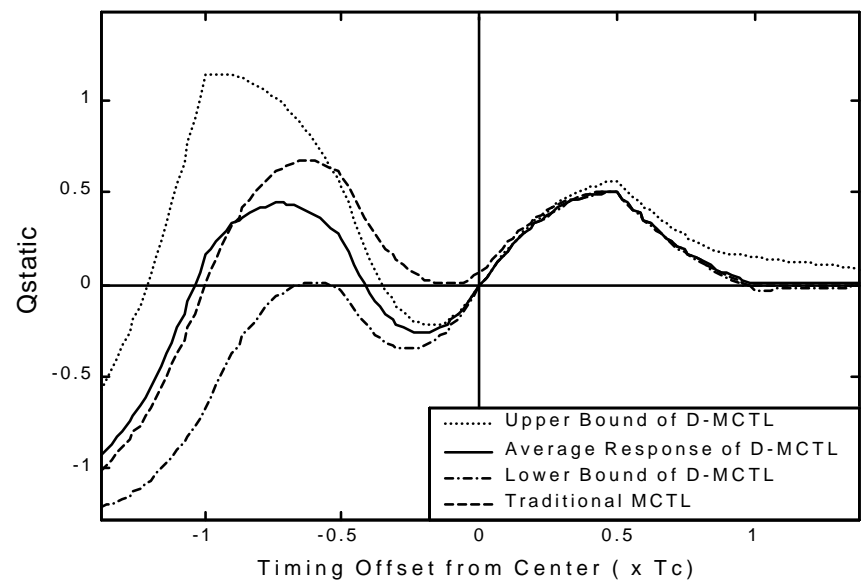

Fig.6 Sequence Length $=63$, no of users $=11, n-f=7 \mathrm{~dB}$, $R_{\mathrm{i}, 1}\left(-T_{c}\right)=-$ ve peak..

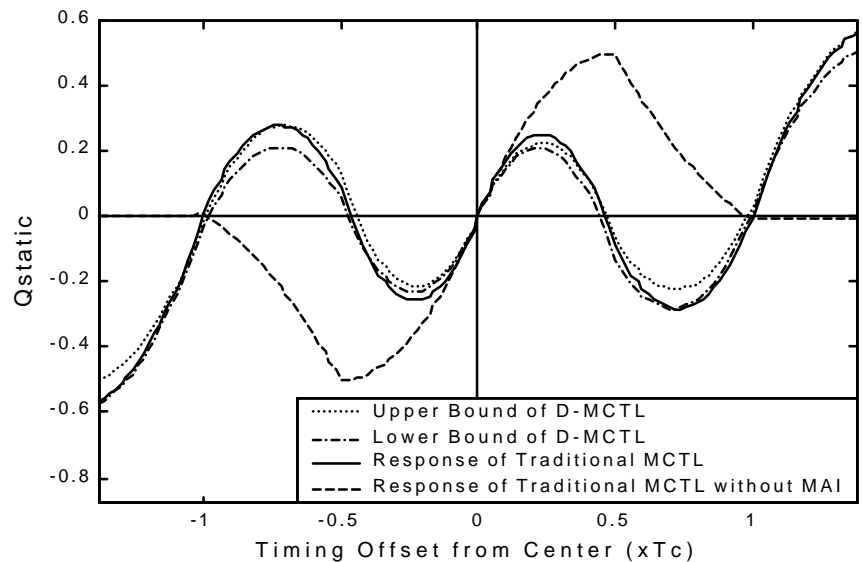

Fig.7 Sequence Length $=63$, no of users $=11, n-f=5 \mathrm{~dB}$, $R_{\mathrm{i}, 1}\left(-T_{C}\right)=+$ ve peak., $R_{\mathrm{i}, 1}\left(T_{c}\right)=+$ ve peak. 